\title{
Aquapathogenesis is the Nature of Human Diseases Dynamic Hirudotests: New Possibilities of Integral Diagnostics of the State of Human Bodies and His Systems - Study of the Dynamism of the Human Aquasystem
}

\section{AI Krashenyuk*}

Academy of Hirudotherapy, St. Petersburg, Russia

*Corresponding Author: AI Krashenyuk, Academy of Hirudotherapy,

St. Petersburg, Russia.
Received: July 20, 2020

Published: September 21, 2020

(C) All rights are reserved by AI Krashenyuk.

\begin{abstract}
The article is devoted to the review of dynamic hirudotests developed by the author and based on well-known biophysical static methods: the Akabane thermopuncture test, the Kirlianography test (Gas-discharge visualization - GDV), and the information-entropy method of academician. G.N. Dulnev with the new device "Eniotron-3". Professor Postnov's S.E. Boundary water and the "SPE-effect" (Sinitsyn-Petrosyan-Elkin) method were used as additional test systems. "SPE-effect" implemented with the original "Aqaphone" device. Keywords: Dynamic Hirudotests; Dynamism; Human Aquasystem
\end{abstract}

\section{Introduction}

For the first time, the discovery of the energy effect of leech therapy (hirudotherapy) on the human body was expressed in our work back in 1993 [1]. In this work, we used the classical static version of the Akabane test (thermal puncture test), which is used by acupuncture specialists from different countries. The essence of the method is that the doctor experimentally conducts a study of the heat sensitivity of the points of entry and exit of the channels (meridians) on the hands and feet of the patient (Figure 1).

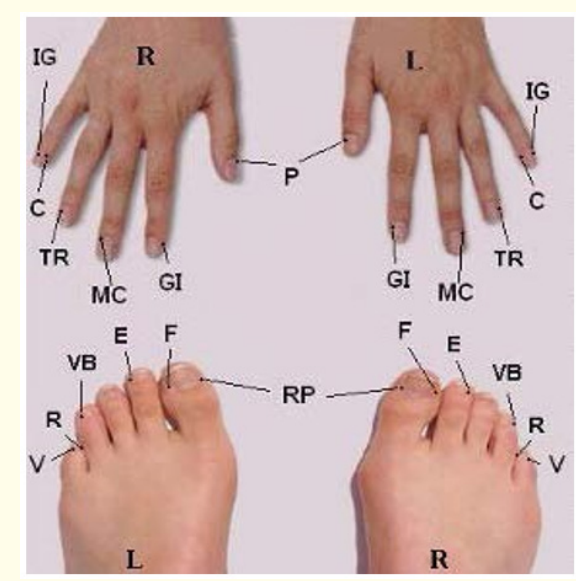

Figure 1: Abbreviated channels names are in French. This corresponds to the designation of the channels in the tables.
Akabane used the smoldering stick of sandalwood for this purpose, which he brought to the experimental point on the skin, at a distance of several millimeters from its surface. In modern devices, point infrared emitters are used for this purpose.

It is well known that dynamic tests are much more informative than static methods. A vivid illustration of this situation is the study of human ECG. If the initial ECG does not provide information about the violation of cardiac blood flow, then the functional load (exercise bike or treadmill) gives the doctor an answer to the localization of the problem area of the myocardium [2]. An even more striking example is the daily monitoring of cardiac muscle function according to Holter.

It turned out that the great diagnostic value of "Load methods" on the heart muscle shows the doctor a vector of the development of the process. Today this statement no longer raises any objections among professionals.

A similar approach was used by us. At the same time, it turned out that Hirudo medicinalis is not only a unique treatment tool but also a subtle diagnostic tool. But the nature of this diagnostic property of Hirudo medicinalis was discovered only in 2001 [3].

\section{Materials and Research Methods}

Test Akabane performed on the Muzhikov's V.G. device "Reflexomaster" [4]. 
Aquapathogenesis is the Nature of Human Diseases Dynamic Hirudotests: New Possibilities of Integral Diagnostics of the State of Human Bodies and His Systems - Study of the Dynamism of the Human Aquasystem

For the objectification of research, the following methods were used:

GDV- analysis using the GDV- Camera instrument by professor Korotkov K.G. [5]. The device allows you to record the glow in real time, in a normal darkened room, and directly on the computer screen to observe changes in the aura of a person or individual parts of his body (for example, fingers). The software allows you to quantify the parameters of images, track the dynamics of the processes occurring in the body. Besides the software package allows you to build a spatial picture of the aura around the patient's body.

In addition, a new device for GDV - "Bio-Well" was used, www. bio-well.com. The 10 fingers of both hands were measured by the "Bio-Well" computerized complex. All GDV- grams were removed without a filter.

The study of information entropy flows was analyzed using the "Eniotron-3" device [6], which was graphically expressed in determining the chaos criterion (Kc) during the study. The device's sensors are technical devices that simulate a double electric layer (DEL) of cell membranes. Diagnosis of the patient's condition is carried out before the hirudotherapy session, immediately after the session, and 30 minutes after the end of the hirudotherapy session.

Resonance-wave studies and diagnostics were performed using the diagnostic complex "Aquafon" ("Telemack", Saratov, www.aquatone.su). The device is based on the fundamental properties of water-containing environments discovered by physicists from Saratov: non-linear dynamics, resonance-wave state and resonant frequencies of own molecular oscillations in extremely high frequencies (EHF) and super high frequencies (SHF) range, "Translational" resonances, resonant radio prostration and transformation of resonant frequencies of $\mathrm{EHF} \rightarrow \mathrm{SHF}$ ("SPE-effect", adequate fractal-cluster model of molecular structure and anomalous properties of water, analogues of quantum effects of Zeeman, Shtark and Faraday in the water in the radio range [7].

As evidence of the nature of the impact of Hirudo medicinalis on the cluster structure of the human aquasystem, we used the Boundary water of professor Postnov S.E. [8]. Boundary water is water with a negative value of redox potential (RP), reaching hundreds of millivolt. This water in a closed container retains its properties, including a negative RP value in the range of $-400 \div 200 \mathrm{mV}$ for at least 6 months.
The technological process of preparing Boundary water is a sequence of effects on the water of several physical factors. Including passing it through a membrane electrolytic cell of a special design with a certain speed, at a certain electric field strength, and KnowHow in the form of "Final packing of water molecules" [RF Patents N. 2479318 and 2615154].

For biologists, microbiologists, virologists, and doctors, the most interesting was the similarity of Boundary water with the properties of water circulating in mammals. It is these properties that made it possible to conduct a series of studies concerning various microorganisms in vitro and animals. It turned out that Boundary Water has a pronounced antiviral activity against human influenza (1:1000) and herpes viruses (1:10 000), as well as mouse encephalomyocarditis virus.

Three types of cancer cells under the influence of the Boundary water drastically slowed down cell reproduction, and then their complete degeneration ensued.

In all these studies, 1 medical leech (ML) was placed on the area of the mucous tissue in the area of Marma Guda (4-finger Marma) [9], until the leech was completely saturated.

Thermopuncture channel diagnostics (Krashenyuk-Akabane test).

We give excerpts and conclusions from our works on this topic (Table 1) [1,6]. Patient K.A.V. turned to our clinic with complaints of exacerbation of hemorrhoids. When examining the pathology zone, a marked exacerbation was noted: increased hemorrhoidal bonds with red blood cell diapedesis, with a mucus deposit. Instead of the expected changes (imbalances) in the channel of the colon (GI) and pericardium (MC), throughout the month we recorded imbalances in the channel bladder (V) (Table 1).

From the diagnostic information content of the Akabane test, such results for 4 protocols (from 11.11 .93 to 09.11.1993) seemed illogical. Because the expected results should be related to channel imbalances (GI) and (MC).

The diagnosis was obvious: exacerbation of hemorrhoids. But an additional examination of this patient revealed a "Silent stone" in the pelvis of the left kidney. And then it became clear why dominated in all 4 protocols (from 11.08 .93 to 11.09.1993) violation (imbalance) in the channel of the bladder (V). 
Aquapathogenesis is the Nature of Human Diseases Dynamic Hirudotests: New Possibilities of Integral Diagnostics of the State of Human Bodies and His Systems - Study of the Dynamism of the Human Aquasystem

An organic lesion of the kidney tissue was discovered, which has not yet been manifested by the clinical sign of "Renal colic", although at the information level this disease has already manifested itself.

The discovered patterns are systemic in nature; they are reproduced in all patients. To date, thousands of patients have been examined, which allows us to make the following conclusions.

Using the Krashenyuk - Akabane hirudotest allows you to: To identify functional disorders of the channels and organs, when the initial state of the channels indicates the imbalance of the channel, and after the Hirudo test we observe the harmony of the channel, then we call this state of the channel a functional (reversible) violation.

Examples: channel (IG), protocol (11.08.1993); channel (VB), protocol (28.08.1993); channel (V), protocol (28.08.1993).

To identify hidden (true) violations of channels and organs, when the initial state of the channels indicates the harmony of the channel, and after the Hirudo test we observe an imbalance of the channel, then we call this state of the channel a hidden (true) violation.
Examples: channel (GI) and channel (R), protocol (11.08.1993); channel (TR) and channel (C), protocol (04.09.1993); channel (P), protocol (11.09.1993).

To identify organic disorders of the channels and organs, when the initial state of the channels indicates disharmony of the channel, and after the hirudotest (session leech in the area of Marma Guda), we observe the preservation of the channel imbalance, and then we call this state of the channel an organic violation.

Examples: channel (V), protocol (11.08.1993); channel (V), protocol (04.09.1993); channel (TR) and channel (V), protocol (11.09.1993).

To identify the dominant violation of the channels and organs, when we statistically identify the most frequently occurring imbalance of channels and organs.

Examples: channel (V), protocols (11.08.1993), (04.09.1993), and (11.09. 1993).

The dynamics of the functional activity of the channels during hirudotherapy in a patient K.A.V., 37 years old $(1$ month of treatment).

\begin{tabular}{|c|c|c|c|c|c|c|c|c|c|c|c|c|c|c|c|c|c|c|c|c|c|c|c|c|c|}
\hline \multirow{4}{*}{$\begin{array}{l}\frac{2}{0} \\
\stackrel{\Xi}{0} \\
\ddot{0}\end{array}$} & \multicolumn{6}{|c|}{11.08 .1993} & \multicolumn{7}{|c|}{28.08 .1993} & \multicolumn{6}{|c|}{04.09 .1993} & \multicolumn{6}{|c|}{11.09 .1993} \\
\hline & \multicolumn{3}{|c|}{ before } & \multicolumn{3}{|c|}{ after } & \multicolumn{3}{|c|}{ before } & \multicolumn{4}{|c|}{ after } & \multicolumn{3}{|c|}{ before } & \multicolumn{3}{|c|}{ after } & \multicolumn{3}{|c|}{ before } & \multicolumn{3}{|c|}{ after } \\
\hline & \multicolumn{6}{|c|}{ hirudotherapies } & \multicolumn{7}{|c|}{ hirudotherapies } & \multicolumn{6}{|c|}{ hirudotherapies } & \multicolumn{6}{|c|}{ hirudotherapies } \\
\hline & D & S & $\begin{array}{l}\text { DS } \\
\text { SD }\end{array}$ & D & S & $\begin{array}{l}\text { DS } \\
\text { SD }\end{array}$ & D & S & $\begin{array}{l}\text { DS } \\
\text { S D }\end{array}$ & D & & & $\begin{array}{l}\text { DS } \\
\text { SD }\end{array}$ & D & $S$ & $\begin{array}{l}\text { DS } \\
\text { S D }\end{array}$ & $\mathrm{D}$ & S & $\begin{array}{l}\text { DS } \\
\text { SD }\end{array}$ & D & $\mathrm{S}$ & $\begin{array}{l}\text { DS } \\
\text { SD }\end{array}$ & D & S & $\begin{array}{l}\text { DS } \\
\text { SD }\end{array}$ \\
\hline$?$ & 19 & 11 & $\begin{array}{l}1,7 \\
2\end{array}$ & 12 & 10 & $\begin{array}{l}1,2 \\
0\end{array}$ & 9 & 7 & $\begin{array}{l}1,2 \\
8\end{array}$ & 9 & & & $\begin{array}{l}1,8 \\
0\end{array}$ & 8 & 12 & $\begin{array}{l}1,5 \\
0\end{array}$ & 17 & 9 & $\begin{array}{l}1,8 \\
8\end{array}$ & 15 & 10 & $\begin{array}{l}1,5 \\
0\end{array}$ & 34 & 8 & $\begin{array}{l}4,2 \\
5\end{array}$ \\
\hline GI & 26 & 17 & $\begin{array}{l}1,5 \\
3\end{array}$ & 25 & 10 & $\begin{array}{l}2,5 \\
0\end{array}$ & 9 & 6 & $\begin{array}{l}1,5 \\
0\end{array}$ & 8 & & & $\begin{array}{l}1,1 \\
4\end{array}$ & 11 & 10 & $\begin{array}{l}1,1 \\
0\end{array}$ & 12 & 9 & $\begin{array}{l}1,3 \\
3\end{array}$ & 12 & 9 & $\begin{array}{l}1,3 \\
3\end{array}$ & 12 & 9 & $\begin{array}{l}1,3 \\
3\end{array}$ \\
\hline MC & 11 & 12 & $\begin{array}{l}1,0 \\
9\end{array}$ & 9 & 8 & $\begin{array}{l}1,1 \\
2\end{array}$ & 9 & 6 & $\begin{array}{l}1,5 \\
0\end{array}$ & 6 & & & $\begin{array}{l}1,2 \\
0\end{array}$ & 8 & 7 & $\begin{array}{l}1,1 \\
4\end{array}$ & 9 & 9 & $\begin{array}{l}1,0 \\
0\end{array}$ & 9 & 8 & $\begin{array}{l}1,1 \\
4\end{array}$ & 9 & 8 & $\begin{array}{l}1,1 \\
2\end{array}$ \\
\hline TR & 10 & 12 & $\begin{array}{l}1,2 \\
0\end{array}$ & 7 & 9 & $\begin{array}{l}1,2 \\
8\end{array}$ & 6 & 6 & $\begin{array}{l}1,0 \\
0\end{array}$ & 0 & & & $\begin{array}{l}1,0 \\
0\end{array}$ & 8 & 8 & $\begin{array}{l}1,0 \\
0\end{array}$ & 6 & 94 & $\begin{array}{l}15, \\
60\end{array}$ & 9 & 19 & $\begin{array}{l}2,1 \\
1\end{array}$ & 7 & 16 & $\begin{array}{l}2,2 \\
8\end{array}$ \\
\hline $\mathrm{c}$ & 12 & 8 & $\begin{array}{l}1,5 \\
0\end{array}$ & 10 & 7 & $\begin{array}{l}1,4 \\
2\end{array}$ & 7 & 7 & $\begin{array}{l}1,0 \\
0\end{array}$ & 5 & & & $\begin{array}{l}1,0 \\
0\end{array}$ & 7 & 8 & $\begin{array}{l}1,1 \\
4\end{array}$ & 14 & 6 & $\begin{array}{l}2,3 \\
3\end{array}$ & 10 & 9 & $\begin{array}{l}1.1 \\
1\end{array}$ & 6 & 7 & $\begin{array}{l}1.1 \\
7\end{array}$ \\
\hline IG & 23 & 8 & $\begin{array}{l}2,8 \\
7\end{array}$ & 6 & 7 & $\begin{array}{l}1,1 \\
7\end{array}$ & 9 & 7 & $\begin{array}{l}1,2 \\
8\end{array}$ & 7 & & & $\begin{array}{l}1,0 \\
0\end{array}$ & 10 & 12 & $\begin{array}{l}1,2 \\
0\end{array}$ & 12 & 13 & $\begin{array}{l}1,0 \\
8\end{array}$ & 9 & 16 & $\begin{array}{l}1,7 \\
7\end{array}$ & 8 & 7 & $\begin{array}{l}1,1 \\
4\end{array}$ \\
\hline 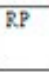 & 6 & 11 & $\begin{array}{l}1,8 \\
3\end{array}$ & 12 & 9 & \begin{tabular}{l|}
1,3 \\
3 \\
\end{tabular} & 10 & 7 & $\begin{array}{l}1,4 \\
2\end{array}$ & 13 & & & $\begin{array}{l}3,1 \\
7\end{array}$ & 6 & 6 & $\begin{array}{l}1,0 \\
0\end{array}$ & 8 & 7 & $\begin{array}{l}1,1 \\
4\end{array}$ & 9 & 11 & $\begin{array}{l}1,2 \\
2\end{array}$ & 11 & 7 & $\begin{array}{l}1,5 \\
7\end{array}$ \\
\hline$F$ & 7 & 8 & $\begin{array}{l}1.1 \\
4\end{array}$ & 12 & 7 & $\begin{array}{l}1,7 \\
1\end{array}$ & 9 & 7 & $\begin{array}{l}1.2 \\
8\end{array}$ & 9 & & & $\begin{array}{l}1,0 \\
0\end{array}$ & 7 & 6 & $\begin{array}{l}1,1 \\
7\end{array}$ & 6 & 6 & $\begin{array}{l}1,0 \\
0\end{array}$ & 10 & 8 & $\begin{array}{l}1,2 \\
5\end{array}$ & 13 & 14 & $\begin{array}{l}1.0 \\
8\end{array}$ \\
\hline E & 8 & 10 & $\begin{array}{l}1,2 \\
5\end{array}$ & 7 & 7 & $\begin{array}{l}1,0 \\
0\end{array}$ & 8 & 8 & $\begin{array}{l}1,0 \\
0\end{array}$ & 7 & & & $\begin{array}{l}1,0 \\
0\end{array}$ & 7 & 8 & $\begin{array}{l}1,1 \\
4\end{array}$ & 10 & 8 & $\begin{array}{l}1,2 \\
5\end{array}$ & 12 & 8 & $\begin{array}{l}1,5 \\
0\end{array}$ & 15 & 23 & $\begin{array}{l}1,5 \\
3\end{array}$ \\
\hline VB & 17 & 13 & $\begin{array}{l}1,3 \\
1\end{array}$ & 18 & 18 & $\begin{array}{l}1,0 \\
0\end{array}$ & 18 & 9 & $\begin{array}{l}2,0 \\
0\end{array}$ & 10 & & & $\begin{array}{l}1,1 \\
2\end{array}$ & 26 & 14 & $\begin{array}{l}1,1 \\
4\end{array}$ & 18 & 17 & $\begin{array}{l}1,0 \\
6\end{array}$ & 16 & 14 & $\begin{array}{l}1,1 \\
4\end{array}$ & 11 & 22 & $\begin{array}{l}2,0 \\
0\end{array}$ \\
\hline $\mathrm{R}$ & 13 & 16 & $\begin{array}{l}1.2 \\
3\end{array}$ & 20 & $\begin{array}{l}14 \\
4\end{array}$ & $\begin{array}{l}7,2 \\
0\end{array}$ & 12 & 10 & $\begin{array}{l}1,2 \\
0\end{array}$ & 13 & & & $\begin{array}{l}1,5 \\
8\end{array}$ & 13 & 11 & $\begin{array}{l}1,1 \\
8\end{array}$ & 24 & 27 & $\begin{array}{l}1,1 \\
2\end{array}$ & 44 & 27 & $\begin{array}{l}1,6 \\
3\end{array}$ & 45 & 43 & $\begin{array}{l}1,0 \\
4\end{array}$ \\
\hline$V$ & 23 & $\begin{array}{l}10 \\
2\end{array}$ & $\begin{array}{l}4,4 \\
3\end{array}$ & 83 & $\begin{array}{l}18 \\
2\end{array}$ & $\begin{array}{l}2,1 \\
9\end{array}$ & 24 & 77 & $\begin{array}{l}3,2 \\
1\end{array}$ & 0 & & & $\begin{array}{l}1,3 \\
3\end{array}$ & 36 & $\begin{array}{l}10 \\
0\end{array}$ & $\begin{array}{l}2,7 \\
7\end{array}$ & 61 & $\begin{array}{l}13 \\
3\end{array}$ & $\begin{array}{l}2,1 \\
8\end{array}$ & 27 & 82 & $\begin{array}{l}3,0 \\
7\end{array}$ & 38 & $\begin{array}{l}19 \\
4\end{array}$ & $\begin{array}{l}9,0 \\
5\end{array}$ \\
\hline
\end{tabular}

Table 1.

Note: $\mathrm{D}$ and S are the right and left branches of channel, respectively;

- Highlighted channels in which violations were detected $(D / S \geq 2.00$ or $S / D \geq 2.00)$.

Abbreviated channel names:

P: Lung; RP: Spleen - Pancreas; GI: Gross Intestine; F: Liver; MC: Pericardium; E: Stomach; TR: Three Heaters; VB: Gall Bladder; C: Heart; R: Kidney; IG: Small Intestine; V: Bladder 
Aquapathogenesis is the Nature of Human Diseases Dynamic Hirudotests: New Possibilities of Integral Diagnostics of the State of Human Bodies and His Systems - Study of the Dynamism of the Human Aquasystem

The conclusions presented in this article were obtained during the examination of more than a thousand patients and allow us to give deeper diagnostic assessments of the condition of the channel (meridional) system of patients.

By "Energy" definition we mean the existence of the phenomenon of a change in the state of energy of "CHI" in Chines traditional terms and concepts.

Following the canons of Chines traditional terms and concepts, it can be suggested that the conditions of the "CHI" energy circulation through human channels change after a leech treatment session.

It should be noted that the above conclusions are true in the case of constant observation of patients in dynamics using the Akabane test.

The use of the Akabane test in combination with hirudotherapy was called in the literature "Hirudotest of Krashenyuk" or "Hirudotest of Krashenyuk - Akabane" [10].

Gas-discharge diagnostics (Krashenyuk-Korotkov-Kirlian test)

Studying th e glow of fingers using the Kirlianography method [11], modified by professor K.G. Korotkov [12], we found an interesting and reproducible fact- a change in the glow area of a person's fingers after a hirudotest. As a rule, hirudotest gave an increase in the area of the glow.

An attempt to explain this phenomenon led us to many years of cooperation with Professor K.G. Korotkov, the author of a whole series of devices that allow real-time (not photographs) examination of patients for diagnostic purposes.

In these studies, it was possible to prove that Hirudo medicinalis is not only a unique treatment tool but also a subtle diagnostic tool that allows the research doctor to identify the area of human pathology.

Although the diagnosis of the disease can be achieved by other methods, the results obtained by Kirlianography were unique.

As evidence of this judgment, we offer an analysis of some clinical cases studied by the author of the publication.

The study of the area of the glow of the fingers in an 11-yearold girl suffering from epilepsy after staging just one leech (Figure 2).

Figure 2 shows the dynamics of changes in the area of the glow of the fingers of an 11-year-old girl before and after setting 1 leech on the Marma Guda region.
The glow of the fingers is a complex picture that belongs to fractals, and its analysis to fractal mathematics. Figure 3 shows the analysis of finger fractals before and after leech treatment in the form of histograms.

As follows from figure 3, the maximum increase in the glow area (S1/S0 ratio) was noted for the thumb. As is known and follows from the Korotkov diagnostic table [5], the thumb reflects the state of the head and brain. An epileptic lesion was found in the patient in the left hemisphere of the brain, and we also see the maximum increase in the glow area (Figure 3) on the thumb.

In figure 4 , we see that the maximum increase in the glow area ( $\mathrm{S} 1 / \mathrm{S} 0$ ratio) was noted for 4 fingers (ring finger). From the Korotkov diagnostic table, the lower pole of the 4-th finger stresses the state of the genitourinary system.

A large amount of experimental material proves that the maximum increase in the glow area always corresponds to the pathology zone.

This means that the maximum increase in the luminous area is a new subtle diagnostic sign of pathology in the context of the Krashenyuk-Korotkov-Kirlian hirudotest.

It is only necessary to compare which of the fingers corresponds to the maximum increase in the area of luminescence after the hirudotest and which organ corresponds to the sectoral topology of the finger - pathology should be sought there.

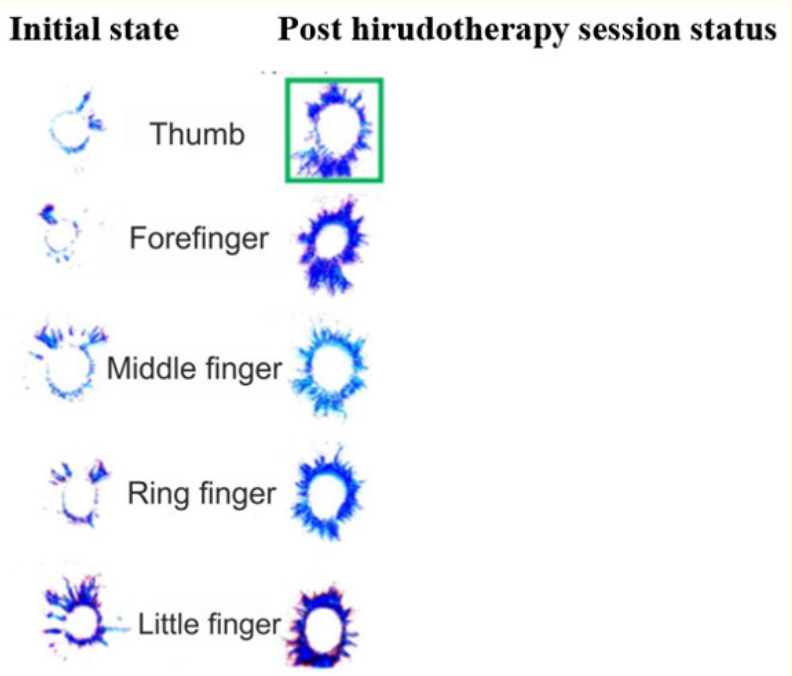

Figure 2: GDV-gram of the patient, 11-year-old Julia. 


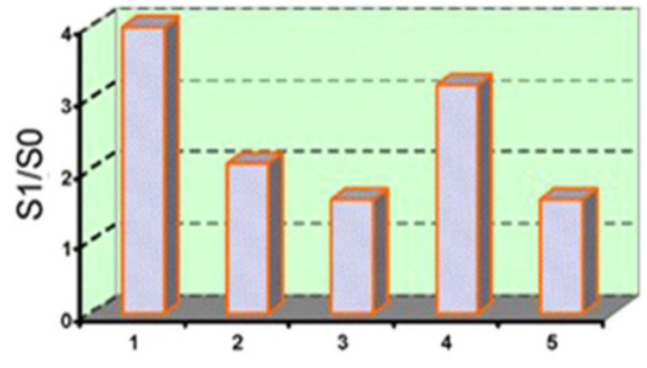

Finger number

Figure 3: GDV - images of fingers before and after a hirudotherapy session and results in their computer processing. Patient Julia A., 11 years old. Designations: along the horizontal axis - finger number; along the vertical axis the ratio of the areas of the glow of the fingers before (SO) and after (S1) session of hirudotherapy. Diagnosis: convulsive readiness of the brain, the focus of excitation in the left half brain according to EEG.

The question also seems natural, what is the nature of such correspondences of the increase in the area of the glow and the pathology of the organ? (Figure 4-7)

The answer to this question was received by us after the discovery of the acoustic (acoustic-electromagnetic) effect of a leech on the aquasystem of the person [3].

Initial state

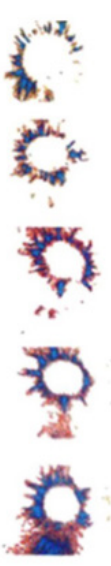

Figure 4: GDV-gram of the patient, 62-year-old man P.V.I.

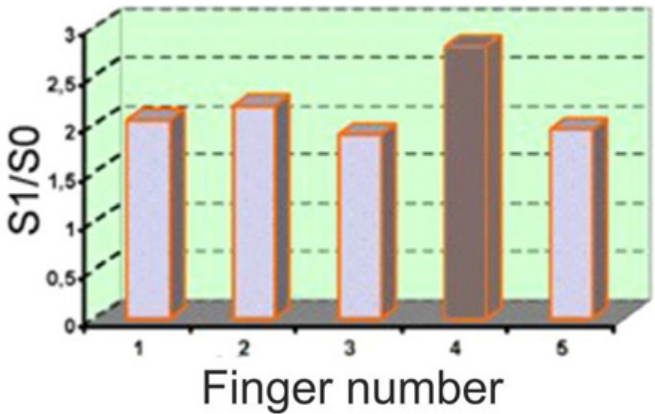

Figure 5: GDV - images of fingers before and after a hirudotherapy session and the results of their computer processing. Patient

P.V.I., 62 years old. Diagnosis: adenoma (hypertrophy) of the prostate according to ultrasound study.

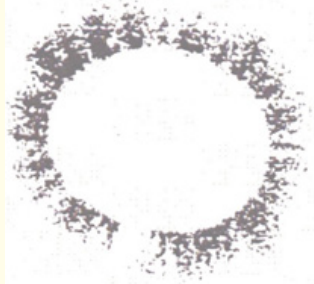

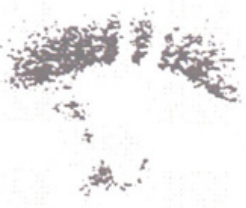

Figure 6: The glow of a human finger and models - artificial capillaries when using the Kirlianography method. Photograph of the glow: A - on the finger; B - on artificial capillaries [14].

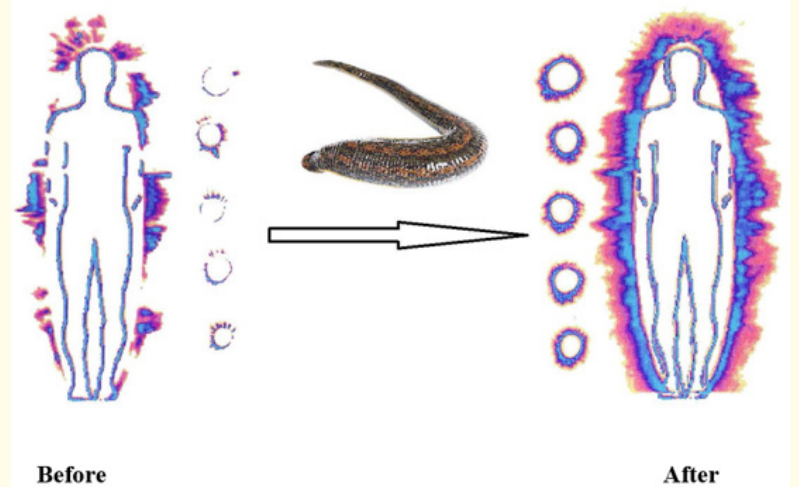

Figure 7: The glow of fingers (analysis result) and body contours human (computer graphics) before and after staging leeches. 
At the fingertips of a person, there is a huge amount of sweat glands. And since all water molecules create a single human aquasystem [13], the change in the glow of the fingers during Kirlianography can be used to judge a person's condition, which was noticed and developed by the Kirlian spouses as a way to diagnose a person's condition [11].

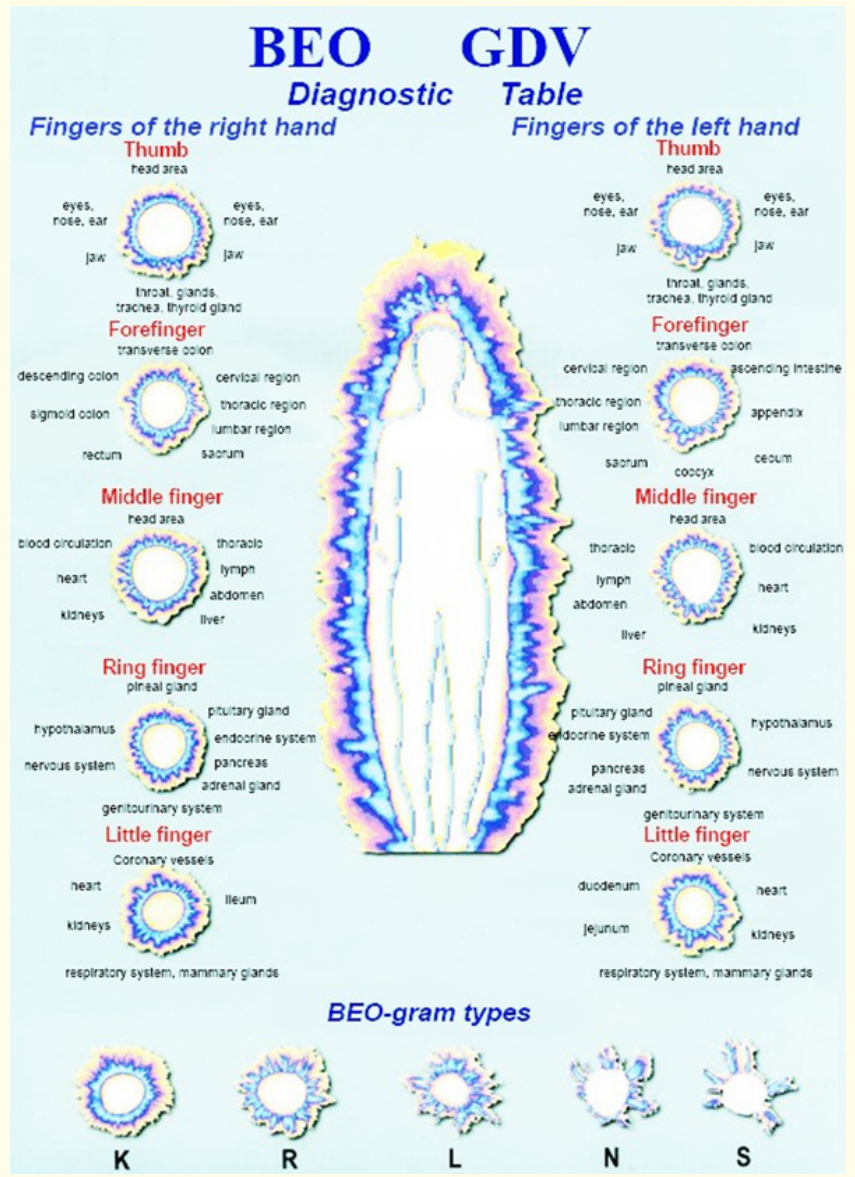

Figure 8: Korotkov diagnostic chart: organ compliance human fingers [6, p.142].

Back in 1996 scientists from Novosibirsk conducted a beautiful and convincing experiment (Figure 6). Figure 6A shows the glow of a finger and figure $6 \mathrm{~B}$ shows model, artificial capillaries - thin tubes with a diameter of $50 \mu \mathrm{m}$, filled with an electrolyte, $0.3 \mathrm{M}$ $\mathrm{NaCl}$ solution. We see on the model a complete analogy of the glow of a finger fragment.

The authors conclude that the observed glow along the contour of the fingers of a person is a corona discharge in the open pores of the sweat glands [14].
These observations are in good agreement with the work of Korkin Yu.V. The author showed that increased sweating by treating the skin surface with acetylcholine increases the area of the GDV signal, while formalin treatment [12].

The result of the dynamic hirudotest Krashenyuk-Korotkov-Kirlian presented in figure 7 .

This figure demonstrates the difference between the static and dynamic versions of the study using Kirlianography. In this case, the doctor was able to understand the nature of the weak glow of the fingers of a 40-year-old man, and prevent vascular catastrophe (acute myocardial infarction or stroke) due to enormous physical overload - the person did not sleep for several nights in a row - transported tourists on the bus during the white nights in St.- Petersburg.

These results indicate the high potential for rehabilitation of this patient, thanks to the performed hirudotest.

Based on a significant number of observations, we were able to identify several patterns that, undoubtedly, are of great diagnostic value.

We examined 240 patients, of whom 56 were men (23.3\%), 184 women $(76.7 \%)$. The results of GDV-graphs in all patients were obtained according to a single, unified method.

A quantitative assessment of these results allowed us to classify patients according to their response to the leech:

- $\quad$ The Superergic Effect is the maximum increase in the luminous area, which is more than $12.0 \%$ concerning the initial state (Figure 7)

- Hyperergic effect: The increase in the luminous area is from $5,1 \%$ to $12,0 \%$.

- Normergic effect: The increase in the area of luminescence is from $1,0 \%$ to $5,0 \%$.

- Hypoergic effect: The increase in the glow area is from $<1,0 \%$ and lower, i.e. we fix a decrease in the magnitude of the glow area.

Based on the results obtained, it can be concluded that the types of patient reactions to leeches occur at different frequencies: most often we encounter a normergic type of response (39\%), a hypoergic type of response - 32\%, a hyperergic type of response occurs in 15\% of cases, superergic type of response - in $14 \%$. 
Aquapathogenesis is the Nature of Human Diseases Dynamic Hirudotests: New Possibilities of Integral Diagnostics of the State of Human Bodies and His Systems - Study of the Dynamism of the Human Aquasystem

Our classification of patients according to the response to hirudotests allowed us to offer doctors a new clinical test, which we called: "The dynamism of the human aquasystem".

In essence, we developed a test in which, as a result of the stimulating effect on the human body of a medical leech, which is based on the phenomenon of acoustic emission, it is possible to qualitatively and quantitatively assess the state of the main functional systems of the body, including the most important one - the Aquasystem of the human body.

Numerous observations of patients during treatment allowed the following patterns to be established. Patients belonging to the group of superergic and hyperergic response gave the fastest clinical effect of improving health status, ceteris paribus.

And patients with a normergic and hypoergic type of response gave slower progress in treatment, and besides, the leeches of these patients in the process of monitoring them often spewed blood and died. These observations allow me to suggest that the blood of patients of this group had signs of toxicity, which allowed us to detect the detoxifying effect of leech therapy. This topic requires special consideration, and possibly a separate article.

As evidence that in Akabane test, kirlianography experiments and Krashenyuk-Dulnev tests, we determine the impact of the leech on the human aquasystem, we used experiments with Boundary water on the same instruments - "Reflexomaster", "GDV-Camera" and "Aqaphone" device.

Informational-entropic definition of the criterion of chaos and order (Krashenyuk-Dulnev test)

Academician G.N. Dulnev created a theory and developed a device for measuring information entropy flows [6].

The sensors of the device were installed on the YUAN points of the left and right branches of the tested channels. The YUAN point (translated from Chinese means "source") is one of the main control points of channels (meridians) in traditional Chinese medicine.

A record of the value of the Chaos criterion (Kc) (the vertical axis of the graph in figure 9) was determined before, during, and after the completion of the hirudotherapy session.

As a result of these experiments, it was shown that the channels (meridians) seemed to "breathe". There is a multidirectional phase change in the magnitude of the flows of information entropy in the channels of the same name and conjugate.
In the channels, as a result of treatment, the level of the chaos criterion (Kc) and the order criterion (Ko) change. The relationship between these values is related as follows:

Ko $=1-$ Kc $[6]$

Moreover, the conjugate channels also turned out to be connected with a change in the value of the chaos criterion (or the order criterion) by the same regularity - they "pulsed" in different phases. Perhaps the most striking it was that the Kc value reached the "Golden Section" level approximately 30 minutes after the leech left (Figure 9).

Those in each session of hirudotherapy, the human information system (channels or meridians) reach the optimal level, the level of the "Golden Section" or the "Golden ratio".

This means that $60 \%$ in this information system corresponds to the order $(\mathrm{Ko}=0.6-0.62)$, and $40 \%$ corresponds to disorder $(\mathrm{Kc}=$ 0.38-0.4), which is apparently) the driving force in the human body to restore order or establish the level of health (Figure 9).

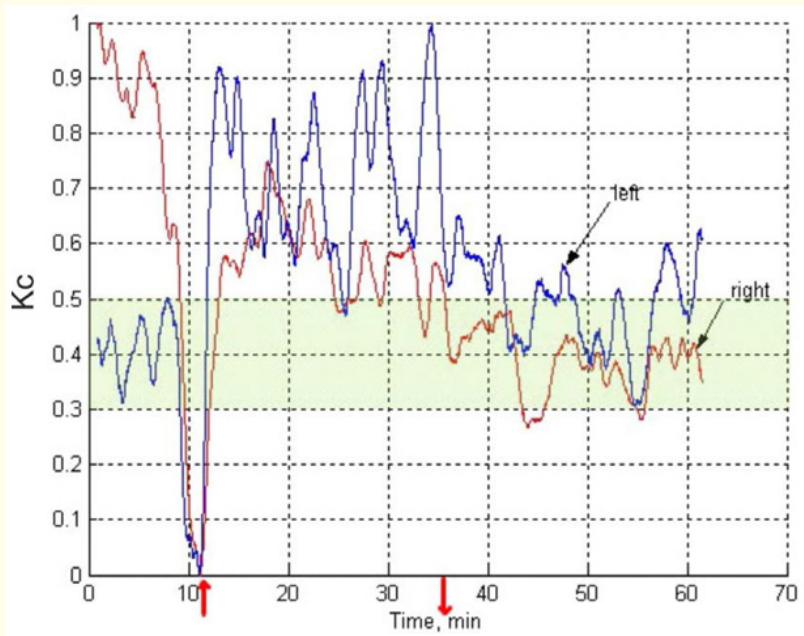

Figure 9: The sensors are mounted on the left and right hand at the point of the YUAN channel of the small intestine. The vertical axis shows the values of the chaos criterion (Kc), along the horizontal axis - the time of observation of the hirudotherapy session. On the 11th minute leech is attached (indicated by arrow),on the 36th-minute leech departed (indicated by arrow).

- The normal zone, Kc $=0.3-0.5$. 


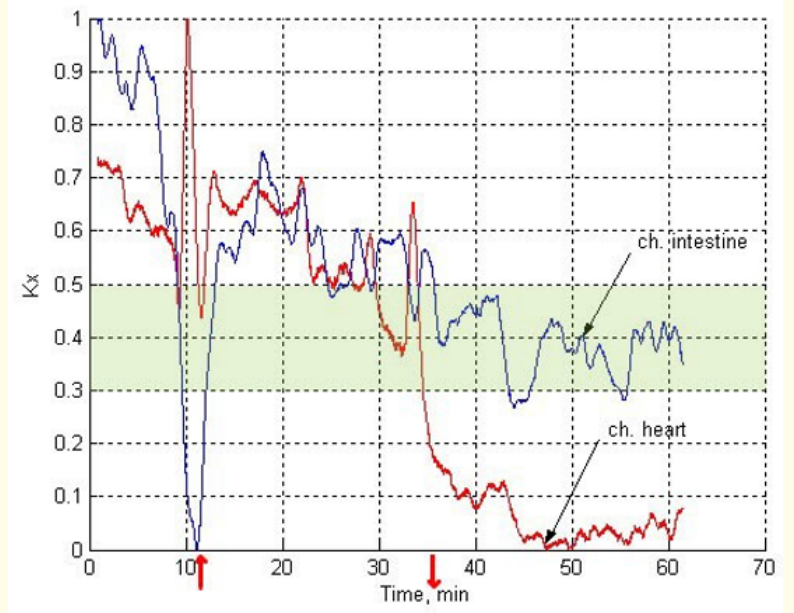

Figure 10: The sensors are mounted on the left hand at the points of the YUAN channels of the small intestine and the heart. The vertical axis shows the values of the chaos criterion (Kc), along the horizontal axis - the time of observation of the hirudotherapy session. On the 11th minute leech is attached (indicated by arrow),

on the 36th-minute leech departed (indicated by arrow).

- The normal zone, $\mathrm{Kc}=0.3-0.5$.

Figure 10 shows a graph of the study of the left branch of the canal of the small intestine and heart. From the graph in figure 10, it follows that both branches of the canal of the small intestine are at the level of harmony ("Golden Section"). At the same time, the left branch of the heart channel is at the level of the maximum value of the order criterion Ko $=1$ (or the minimum value of the chaos criterion $-\mathrm{Kc}=0$ ).

This is a study of a 24-year-old woman suffering from increased thyroid function. Syndrome complex "Thyrotoxic heart" is well known - damage to the cardiovascular system in these patients, thyrotoxic myocardiopathy. If you use the sensors-sensors of the "Eniotron-3" devise for all human channels, it is possible to obtain a scenario for the development of its possible pathology in the future. The result of these studies has made possible a fundamentally new definition of the level of health: "Health is the ability of the body's control systems, based on self-regulation mechanisms, to optimize the levels of information entropy flows through various information channels or tissues to the "Golden Ratio", or "Golden Proportion", and maintain this state for a certain period. This period is determined by the life expectancy of a person".

Resonant-wave studies of the impact of Hirudo medicinalis on the human aquasystem with the help of the Diagnostic Complex "Aquaphone".
On figure 11-13 presented the results of the effect of Hirudo medicinalis on the nasal septum and the patient's spine. The "Aquaphone" diagnostic complex implements the principle of converting the resonant frequencies of the microwave EHF $\rightarrow$ SHF ("SPE" - effect"). With this complex, we were able to prove the impact of $\mathrm{Hi}$ rudo medicinalis on the cluster structure of the human aquasystem. Changes in the frequency characteristics of the human aquasystem are reflected in different colors in the presented images of human anatomical zones.

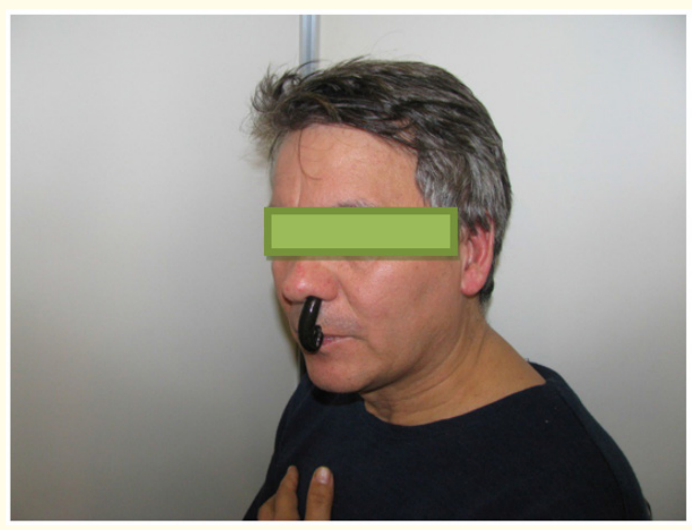

Figure 11: Placing a leech on the nasal septum on the left.

Evaluation of the intensity of stimulated microwave radiation by the radio-electronic complex "Aquafon". The area of the face on the left before and after setting the leech.

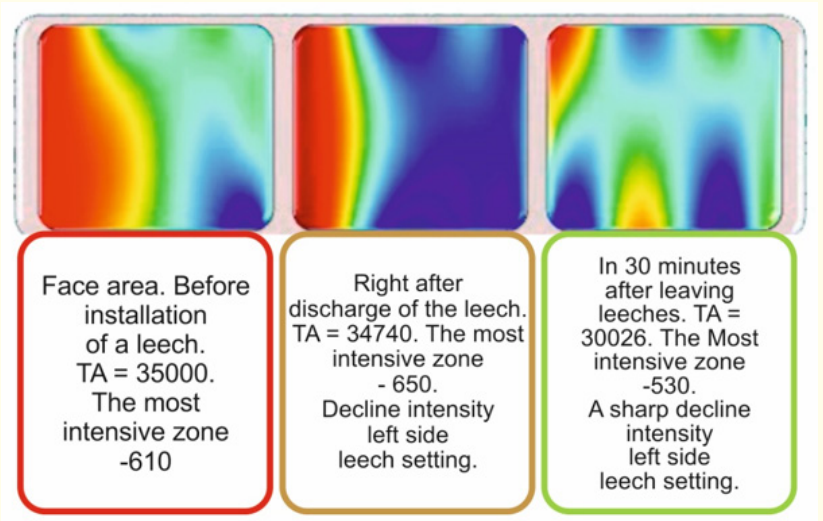

Figure 12: Evaluation of the intensity of stimulated microwave radiation by the radio-electronic complex "Aquafon". The area of the face on the left before and after setting the leech. The intensity of the radiation is determined by the color, higher: burgundy and red, less intense: dark blue and blue. 
Aquapathogenesis is the Nature of Human Diseases Dynamic Hirudotests: New Possibilities of Integral Diagnostics of the State of Human Bodies and His Systems - Study of the Dynamism of the Human Aquasystem

The burgundy and red color reflects the area of inflammation of the left sinus, dark blue and blue color reflects the aquasystem of the sinuses in normal, after exposure to the leech.

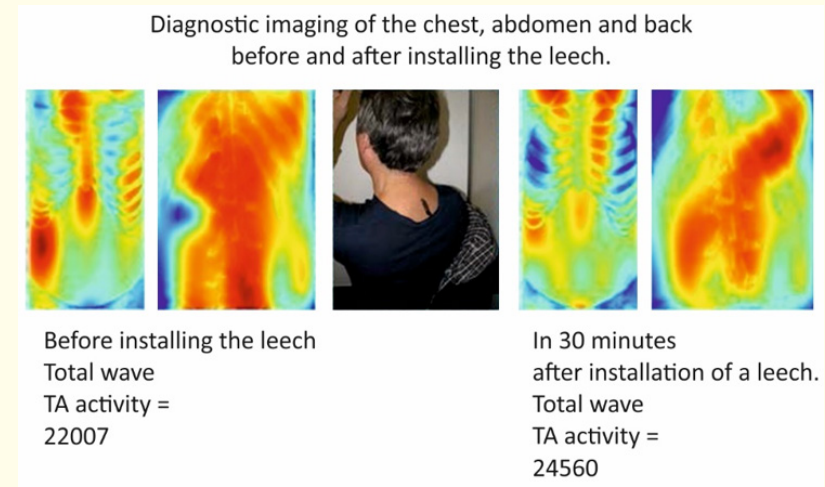

Figure 13: Evaluation of the intensity of stimulated microwave radiation by the radio-electronic complex "Aquafon" [22].
The results of the Boundary water experiments.

The results of the Akabane test when taking boundary water (Table 2)

The $0.4 \mathrm{ml}$ of Boundary water was injected into the oral mucosa to the volunteers and an objective assessment of their condition was carried out by the aforementioned methods.

The results of the study of the impact of the Boundary water by the Akabane method are presented in table 2. From table 2 it follows that after the impact of the Boundary water by the volunteer U.A.F. significant changes have occurred in the canal of the stomach (E), the channel of the kidneys (R), and the channel of the bladder (V). It is well known from the canon of Chinese traditional medicine that it is the channels of the kidneys and bladder that regulate the flow of all body fluids [16].

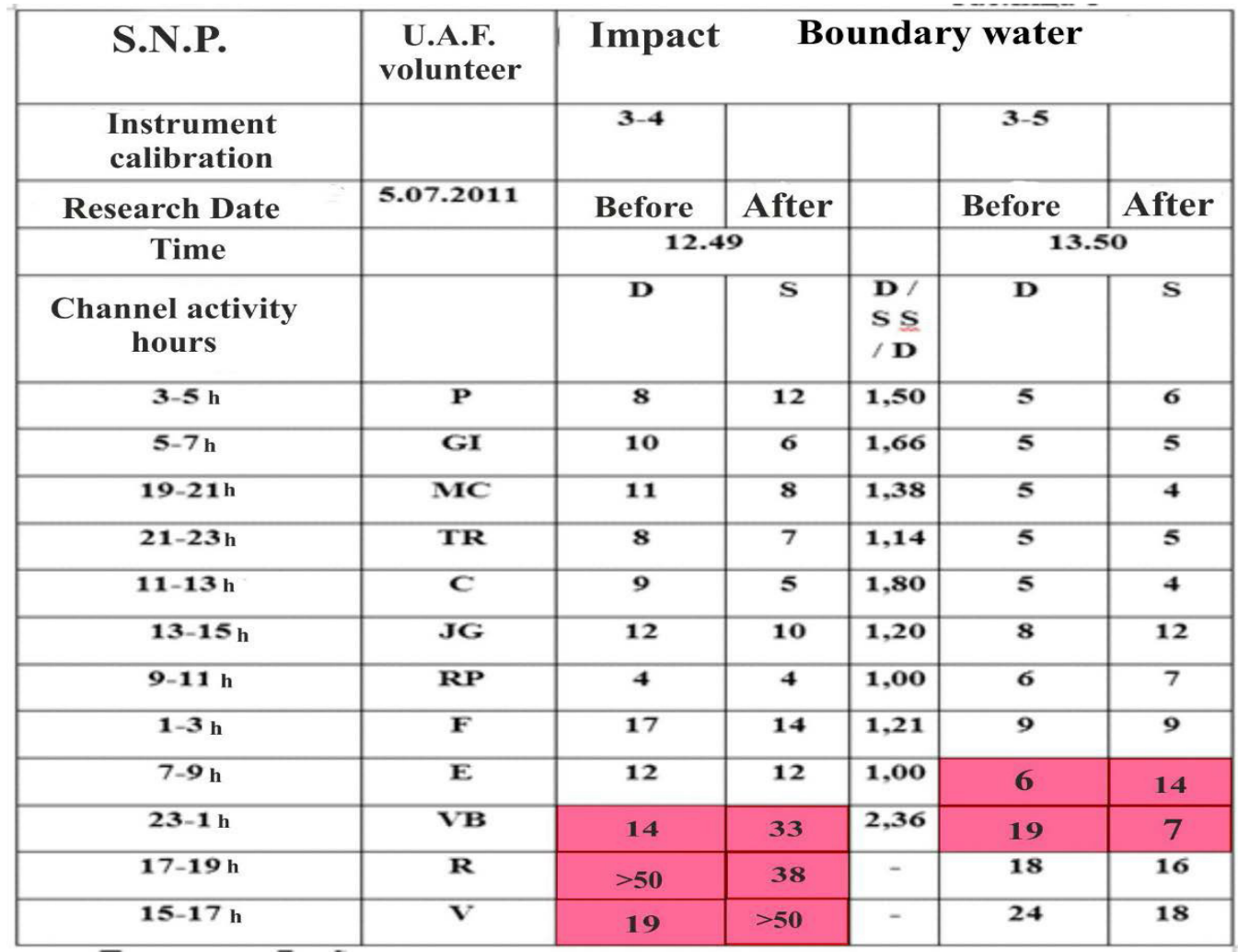

Table 2

Note: D and S are the right and left channel, respectively;

- Highlighted channels in which violations were detected (D/S $\geq 2.00$ or $S / D \geq 2.00)$. 


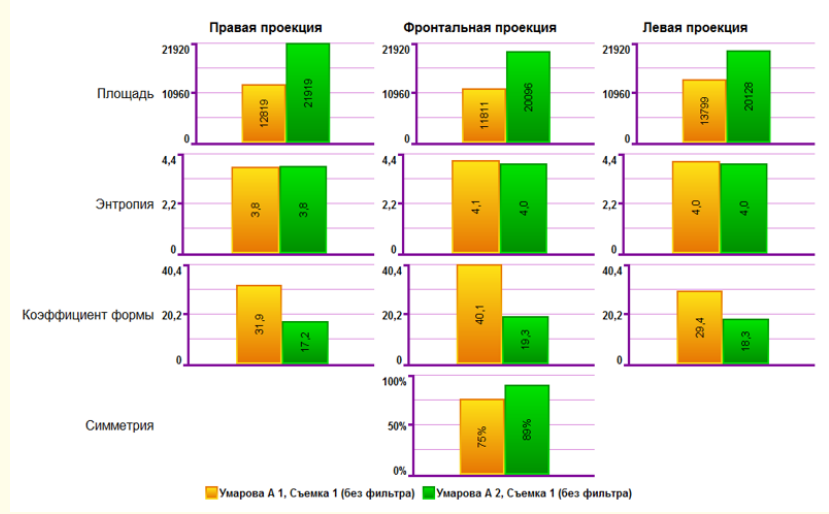

Figure 14: Changes in GDV luminescence parameters in volunteer U.A.F. before and after drinking the Boundary water. Left columns (yellow) - the state before, the right columns (green) - the state after the impact of the Boundary water on the human body.

Changes in the parameters of GDV glow in the U.A.F. volunteer before and after the reception of Boundary water are presented in figure 8. As it follows from figure 14, the area of glow in the frontal projection of the volunteer U.A.F. increased by $70 \%$ (!) after exposure to Boundary water in the record without a filter, and the symmetry ratio increased from $75 \%$ to $89 \%$. This is comparable to the results of the work [15], although in our experiment the Boundary water did not drink water, but received it by spraying it on the mucous mouth.

The data obtained with the "Eniotron - 3" device are shown in figure 15.

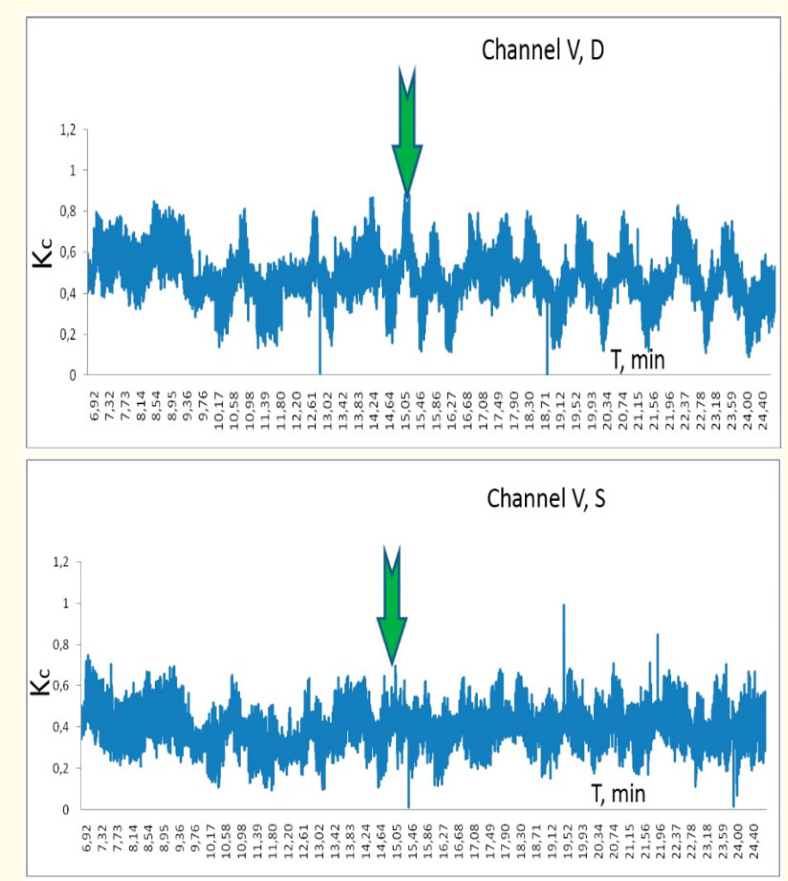

Figure 15: Dynamics of changes in the chaos criterion (Kc) under the influence of the Boundary water on the volunteer U.A.F.

Legend on the graph: horizontally - the experiment time in minutes, vertically - the value of the chaos criterion (Kc). The arrow indicates the time of exposure to the Boundary water volunteer; M.P. - the channel of the bladder (V). D - right branch of channel V. $\mathrm{S}$ - left branch of channel V.
After the influence of Boundary water, changes in the nature of the oscillations of the flow of information entropy (Kc values) are almost instantly visible. They acquire the form of regular harmonics, which is especially noticeable on the right branch of the D channel of the bladder (V) (Upper part of figure 15).

\section{The Discussion of the Results}

The analysis of the experimental materials presented in this article indicates a common reaction mechanism for all hirudotests. Such a general mechanism, in our opinion, is the sensitivity of all three methods to changes in the cluster state of the aquatic system of the human body on the effect of Hirudo medicinalis.

The term "Aquasystem" was first proposed by a professor Slesarev V.I. [13]. The aqua system is a combination of all body fluids: intracellular water, extracellular water, blood, lymph, cerebrospinal fluid, urine, sweat.

As evidence of the nature of the impact of Hirudo medicinalis on the cluster structure of the human aquasystem, we used the Boundary water of professor Postnov S.E. [8]. The positive results and the absence of side effects made it possible to register Boundary water as a food supplement called "Aqua Bonus".

With the help of a new modification of the apparatus of professor Korotkov K.G. "Bio-Well” [17], we were able to test the dynamics of changes in the energy of the human aquasystem after exposure to Hirudo medicinalis and Boundary water (Figure 16 and 17).

The results were impressive. In figure 16, we see a series of waves during and after exposure to a leech, more pronounced during the action of a leech.

In figure 17 we see, after exposure to Boundary water, the periodic responses of the human aquasystem with an interval of about 10 minutes.

This indicates that the main point of application of the acoustic- electromagnetic effect of Hirudo medicinalis is the human aquasystem.

As a result of this work, we obtained several new experimental data that allow us to suggest that the "final point of application" of various medical technologies may be the effect on the structure of water in the human body.

This hypothesis also follows from numerous experiments using the gas- discharge visualization method (GDV- method or Kirlian effect) when studying the effect of the hirudotherapy method on the human body. Why does the picture of the corona discharge on the fingers change (the glow area increases) when using the hirudotherapy method?

The discovery of the wave effect of hirudotherapy [3] helps to answer this question.

For the first time, the radio properties of a leech were experimentally recorded by a piezoelectric transducer during its bloodsucking in 2001 [3]. 
Aquapathogenesis is the Nature of Human Diseases Dynamic Hirudotests: New Possibilities of Integral Diagnostics of the State of Human Bodies and His Systems - Study of the Dynamism of the Human Aquasystem

Using a piezoelectric sensor, it was possible to isolate a signal from continuous noise in the range $25-250 \mathrm{kHz}$ due to stochastic resonance between the leech and piezoelectric sensor signals. As a result of the Fourier transform of the received signal into harmonic oscillations, its spectrum was revealed. Since the piezoelectric sensors are sensitive to both acoustic and electromagnetic radiation, it was not possible to establish its nature, and it was assigned to the acoustic signal, calling it a "song" of leeches. This is only a small part of a large symphony performed by a leech!

The presence of radio properties in water and living organisms justifies their ability to interact according to the resonantwave mechanism.

It is this mechanism that explains the remote, that is, without direct contact, the interaction of water and living systems with each other.

A leech in the process of bloodsucking generates up to 500 550 pulses in the ultrasonic range, which can affect the structural changes of intracellular and intercellular water (the state of associates and clusters) [18].

Both leeches and Boundary water act on the human aquasystem in a similar way, initiating the dissociation of water with the formation of free radicals. The dissociation of the water molecule into ions generates energy of $55.84 \mathrm{~kJ} / \mathrm{mole}$, water dissociation with the formation of free radicals and active forms of oxygen species under the influence of initiation (acoustic-electromagnetic fields) generates the energy of $+542.84 \mathrm{~kJ} / \mathrm{mol}$, i.e. almost 10 times more.

This leads to an increase in the area of the glow of the fingers after leech treatment (Krashenyuk-Korotkov-Kirlian test), changes in the condition of the channels(Krashenyuk-Akabane test) and a change in the chaos criterion - Kc (Krashenyuk-Dulnev test).

Our experiments also support this assumption with professor P. Semikhina [19]. In this work, based on the dielcometric method, it was shown that staging a leech to the frog Rana temporaria significantly changes the cluster structure of intracellular water in these animals, and the sizes of clusters of intracellular water in frog tissues change (Figure 16).

Besides, professor Semikhina L.P. proved that pathologically altered tissue (for example, a tumor) contains an altered cluster structure of the aquasystem [20].

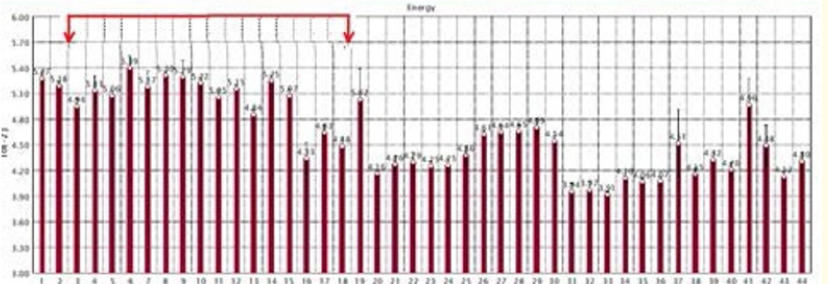

Figure 16: The dynamics of changes in the energy indicator in the session hirudotherapy and after leaving the leech for 1 hour. Arrows indicate the beginning and end of the session hirudotherapy. Patient S.A.E.

A similar conclusion follows from the work of other authors of an earlier work based on the Kirlian effect [21].

The authors of this work suggest a method of early diagnosis of cancer, based on the method of Kirlianography (GDV).

It was found that the values of blood clotting cancer patients reliably exceed the parameters of healthy donors. The authors conclude that the difference in gas discharge parameters (geometric, bright, spectral characteristics) of cancer and donor blood indicates the presence of some factors in the cancer blood that affect the characteristics of the gas discharge glow, which can serve as a basis for early diagnosis of cancer by the characteristics of gas discharge of blood samples.

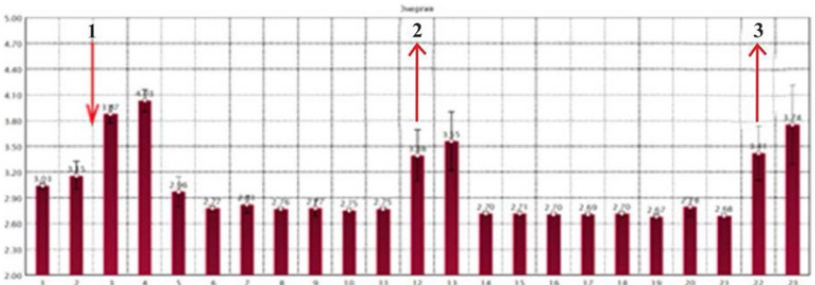

Figure 17: The dynamics of changes in energy after exposure. Boundary water at 3 minutes into the nasal mucosa. A patient D.A.I. The arrows indicated: 1 - effect on the nasal mucosa Boundary water; 2 and 3 - resonances of an aquasystem of an organism on the effects of Boundary water.

Similar but larger studies were carried out by the Indian authors [22]. 
Aquapathogenesis is the Nature of Human Diseases Dynamic Hirudotests: New Possibilities of Integral Diagnostics of the State of Human Bodies and His Systems - Study of the Dynamism of the Human Aquasystem

The quantitative analysis showed that the density of image lighting in healthy patients was significantly less than in patients even the first stage. The density of image lighting increased in patients with an increase in the degree of disease. The use of "water test" (handwashing) led to the disappearance of the light with its subsequent gradual restoration.

At the same time, in healthy patients the degree of reduction was stronger, and the recovery time is longer than in patients.

In our opinion, the Boundary water system at the fingertips reflects the state of the internal human aquasystem, and in cancer. Moreover, it varies with the fact that it changes with the degree of development of cancer.

A significant proof of the points of view expressed is the outstanding work of mathematician and physicist professor Petrosyan V.I. [7].

In the monograph on the principle of material-wave dualism of Nature, the aqua-phase-wave paradigm of life is discussed in normality and carcinogenesis. The body's water environment is in a resonant-wave (RW) state and is an electromagnetic (EM) system of life as a component of homeostasis.

Under normal conditions, there are two alternative phases of water: hexagonal and cubic in RW at frequencies - hexagonal phase, GHz: 50.3, 51.8, 0.985, 0.1 and cubic phase, GHz: 51, 0.99 .

Phase structure and resonant radio waves form the basis of life and they are defined by Formats of life: Format I in normal and Format II in carcinogenesis. The Formats are in a state of reciprocal RW transformations by the impact of resonant radio waves of the alternative phase.

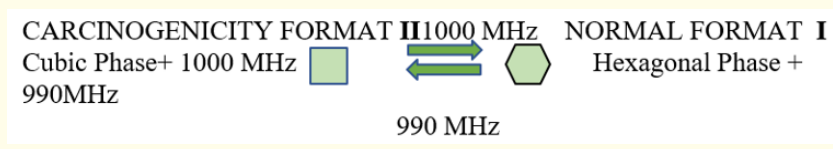

Figure a

The effect of radio wave transformation of carcinogenic Format II in Form at I norm by the influence of resonant radio waves of the norm is laid in the principle of therapy, and the reverse effect of the transformation of Format I norm into carcinogenic Form at II by exposure to resonant carcinogenic radio waves is more environmentally friendly factor carcinogenesis.
Thus, Cancer is not a disease, but a carcinogenic format of life. Radio-electronic complexes and methods of non-load-heavy RW diagnostics and therapy have been developed, where in Format I diseases are manifested in changes in the intensity of RW condition and radio radiation, and carcinogenesis is manifested in a change in resonance frequency.

The main feature of $\mathrm{RV}$ therapy in carcinogenesis is not the loading conversion of carcinogenic cells into norm cells and compensation for the negative effects of chemotherapy and radiotherapy methods.

RW diagnostic and therapy methods are compatible with the methods used in oncology increase their effectiveness and appear to be promising for use in clinical practice and ecology.

It is shown that in the biosphere there are man-made carcinogenic radio waves.

Environmental problems of man-made carcinogenic biosphere diophon are solved by the opposition of radio waves to norms and crusts of sanitary rules and norms of man-made electromagnetic sciences.

Based on the presented concept, Hirudo medicinalis's acoustics and radio radiation can be considered as a preventive and therapeutic factor in the treatment of cancers.

Still, the question remains, why does GDV analysis show us an increase in the area of luminescence after the effects of Boundary water on the human body?

The term "Free radicals" has a quantitative characteristic. Currently, quantitatively determined by gas discharge visualization (GDV).

This happens as follows: the studied object (water, boundary water on the skin of the fingers) is placed in an electromagnetic field. Electrons flying out of an object under its action initiate a corona discharge in the gas, the intensity of which is recorded. Thus, choosing a standard, one can compare the studied objects with each other, since the more electrons participate in the development of the corona discharge, the more intense the glow. For example, a person's condition before and after a hirudotherapy session.

A change in the glow of a person's fingers before and after a hirudotherapy session means that the structure of intracellular water in the epithelial cells of the fingers has changed, the number of free radicals has increased in it. 
So, the structure of intracellular water in the whole body has changed!

- $\quad$ Therefore, the GDV method, as a measurement method from a physical point of view, developed by professor Korotkov K.G. [5] turned out to be a tool which is possible to physically register the state of free radicals in aqueous media.

- We got a tool with which you can study the state of living and nonliving matter, a living organism, and the effectiveness of impacts on it, for example, using

- Boundary water or various medical technologies, in particular, hirudotherapy. As a result of the obtained experimental data, there is also an idea that the "Eniotron-3" device's sensor system is not only a technical device (sensor) but also layers of polarized water adjacent to the base of this sensor.

- Under the technical device, there may be many layers of polarized water (many millions of layers of water dipoles). Since this water is an aquasystem, it will also have the properties of Boundary water. And it is this water that will be the physical (material) carrier of the information that we want to transmit to a person using this or that medical technology, or the impact of music, theater, dance, meditation, prayer. And even the transmission of information remotely can be recorded using a sensor device such as an aqua-radio system with its ability to radiate and transmit acoustic and radio signals.

The essence of the above can be demonstrated in figure 15 .

In our experiments, using a sensor, which is a thermoelectric module with a double electric layer, water dipoles must be polarized in a special way relative to the surface of the sensor. And this feature should give, in our opinion, additional properties. Future experiments in this direction will clarify our assumption.

Information sensor operation model

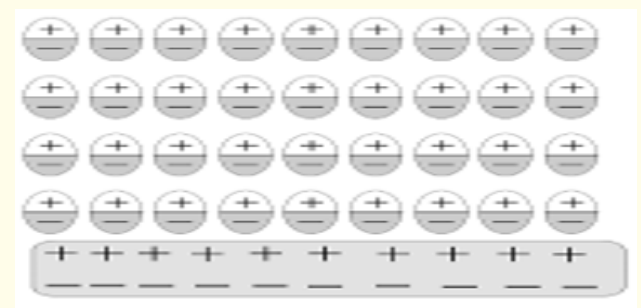

Figure 18: Hypothetical model of the sensor (technical thermoelectric module devices with dual electric layer-DEL) and oriented (polarized) dipoles of the aquasystem of the body.
Our results allow us to expand the interpretation of the GDV analysis of the human condition. They allow us to talk about the possibility of changing the aquasystem with various effects on humans, i.e. evaluate the degree of their positive or negative impact.

This conclusion is consistent with the hypothesis of the phenomenon of "Aquacommunication", developed by Russian scientists Slesarev V.I. and Danilov A.D. [13].

Thus, this is consistent with the conclusions of our ideas that in the field of pathology of any organ the cluster structure of the aquatic system has been changed.

Under the influence of the acoustic-electromagnetic radiation of a leech, a resonance effect occurs in the altered aquasystem and the birth of free radicals in the organ aquasystem.

These phenomena lead to a change in dynamic hirudotests: Krashenyuk- Akabane, Krashenyuk-Korotkov-Kirlian, and Krashenyuk-Dulnev.

Summing up the work related to informational - entropic, acoustic (wave), negentropic and aqua-structuring effects of hirudotherapy, it should be noted that they introduce a fundamentally new understanding of the mechanisms of action of Hirudo medicinalis, the main point of application of which is the body's AQUASYSTEM [23].

\section{Conclusions}

- Aquapathogenesis - is the nature of human diseases and all living things

- Akabane test, GDV (Kirlianography) method, "Eniotron-3" device, "Aqaphone" device can be used to study the body's aquasystem of a person.

- The hirudotests of Krashenyuk-Akabane, Krashenyuk-Korotkov-Kirlian, Krashenyuk-Dulnev can be used to study the dynamism of the human aqua system and predict the clinical prognosis of patient treatment.

- The hirudotest of Krashenyuk-Akabane significantly improves the objectivity and reliability of the diagnostic information of the thermopointur test.

- The hirudotest Krashenyuk-Korotkov-Kirlian, it is possible to determine the change of the aquasystem in the area of the pathologically altered organ, which allows to significantly increasing the diagnostic value of the Kirlianography method, including cancer. 
Aquapathogenesis is the Nature of Human Diseases Dynamic Hirudotests: New Possibilities of Integral Diagnostics of the State of Human Bodies and His Systems - Study of the Dynamism of the Human Aquasystem

- The hirudotest Krashenyuk-Korotkov-Kirlian allows determining the dynamism of the patient's aquasystem and predicting the results of the time of the pronounced positive therapeutic effect of treatment.

- The hirudotest Krashenyuk-Dulnev allows, on the basis of the definition of physical thermodynamic parameters - the criterion of chaos and the criterion of order to get a diagnostic characteristic of the state of human organs and systems and for the first time in the world on the basis of these values to give definitions of human health.

- Hirudotherapy-the complex effect of natural pharmacotherapy, physiotherapy (resonance-wave effect), Aqua therapy on the human or animal organism, which determines its unique therapeutic effectiveness.

\section{Bibliography}

1. Krashenyuk AI and Krashenyuk SV. Diagnostic value of the test Akabane in hirudotherapy. Successes of hirudology. Materials III Conferences of the Association of Hirudologists. Ed. Ph.D. V.V. Ptushkin. M., (1993): 42-43.

2. Mikhailov VM. "Heart rate variability: practical experience application". Ivanovo State Medical Academy. Ivanovo (2002): 288.

3. Krashenyuk AI and Frolov DI. Interpretation of nature energyinformational effect of hirudotherapy based acoustic phenomenon. Abstracts of the V International Congress of Bioelectrography. Science. Information. Consciousness. St.-Petersburg (2001): 89-90.

4. Muzhikov VG. "Theory and practice of thermopuncture channel diagnostics and treatment". St.- Petersburg, (2000): 268.

5. Korotkov KG. Fundamentals of GDV bioelectrography. St.-Petersburg (2001): 73.

6. Dulnev GN and Krashenyuk AI. "From Synergies to information medicine". St.-Petersburg, Institute of Biosensory Psychology (2010): 166.

7. Petrosian VI. "Aqua-phase-wave nature cancerogenesis. Carcinogenic and therapeutic radio waves of the biosphere". Riga Latvia European Union: Lambert Academic Publishing (2018): 96.

8. Postnov SE. "Boundary water in a living organism". M., ed. "Elf IPR". 71.
9. Frawley D., et al. Marmatherapy: energy points Yoga and Ayurveda". Rostov-on-Don, Phoenix (2004): 319.

10. Krashenyuk AI., et al. Bioelectrographic correlates of hirudotherapy. I International Scientific Congress "NEUROBIOTELECOM- 2004". "Infocommunication technologies and radiosystems in medicine, neuroscience and education. Collection of scientific papers, St.- Petersburg, Publishing House "POLYTECHNICA", St.-Petersburg (2004): 145-147.

11. Kirlian SD and Kirlian VH. "Photographing and visual observation at high- frequency currents". Journal of Scientific and Applied Photography and Cinematography 6.6 (1961): 397-403.

12. Korotkov KG. Kirlian effect. Ed. “Olga”, St.- Petersburg (1995): 218.

13. Slesarev VI and Danilov AD. Water! Aquacommunication and aquaclatration is the basis of a new paradigm of medicine. Fourth International Scientific Congress "NEUROBIOTELECOM - 2010". Infocommunication technologies in science, healthcare, and education. Collection of scientific papers, St.-Petersburg, December, 7-10, 2010. Ed. “Teledom”, St.-Petersburg (2010): 274-277.

14. Sankin GN and Teslenko VS. "Study of electric discharge in air with a capillary electric electrode". Letters to the JTF 24 (1996): 49-53.

15. Volkov AV., et al. Registration by the method of HRV action of electrochemically polarized water (A-water, Boundary water) on the human body. Science. Information. Consciousness. The collection of works of the XI International Congress on GDV bioelectrography. St.-Petersburg, 2007, 76-77.

16. Chinese Acupuncture and Moxibustion. Chief editor Cheng Xinnong. Foreign Languages Press, Beijing (1987): 234.

17. Krashenyuk AI., et al. Study of the in influence Of Diagnostic ultrasound of the Human Aqua-System with "Bio-Well" Device". Journal of the Science of Healing Outcomes 9.36 (2017): 5-15.

18. Krashenyuk AI., et al. Changes in the structure of intracellular water - the biophysical basis of energy information processes metabolism in humans and animals. V International Scientific Congress "NEUROBIOTELECOM- 2012". Infocommunication technologies in innovations, medical and biological and technical sciences. Collection of scientific papers, St.-Petersburg, December 7-10, 2012 Publishing house "Teledom", St.-Petersburg, 101-111. 
19. Semikhina LP and Krashenyuk AI. Aqua structure effect hirudotherapy. In the book: "Science. Information. Consciousness". Abstract XIV International Congress on Bioelectrography. St.Petersburg 3-4 (2010): 33.

20. Semikhina LP. "Dielectric and magnetic properties of water in water solutions and bioobjects in weak electromagnetic fields". Tyumen State University (2006): 164.

21. Hurwitz BL., et al. "Shorty Conceptual Approach to Early Cancer Diagnosis”. From Kirlian's effect to Bioelectrography. Ed. “Olga”, St.-Petersburg (1998): 125-132.

22. Chowhan RS., et al. Comparison of bio-electrographic images of cancer patients and healthy patients. Ed. "Olga”, St.-Petersburg (1998): 133-140.

23. Krashenyuk AI and Krashenyuk SV. "Hirudotherapy and the body's aquasystem: a new understanding of Ayurvedic technology". Asclepeion 1-4 (2014): 35-39.

\section{Assets from publication with us}

- Prompt Acknowledgement after receiving the article

- Thorough Double blinded peer review

- Rapid Publication

- Issue of Publication Certificate

- High visibility of your Published work

Website: www.actascientific.com/

Submit Article: www.actascientific.com/submission.php Email us: editor@actascientific.com

Contact us: +919182824667 